\title{
The application of a new mean value theorem to the fractional parts of polynomials
}

\author{
by
}

Trevor D. Wooley (Ann Arbor, Mich.)

1. Introduction. The new mean value estimates provided by recent advances in Waring's problem (see, in particular, $[9,10,11,14]$ ) may plainly be applied to improve current estimates for $\left\|\alpha n^{k}\right\|$, the distance from $\alpha n^{k}$ to the nearest integer (which is intimately related to the fractional part of $\alpha n^{k}$ ). The rather recent arrival of these new estimates has apparently left insufficient time for workers in the field to record in the literature the methods required to cultivate the ensuing bounds on the fractional parts of polynomials. In this paper we intend to fill this lacuna in the literature, at the same time providing a technical improvement to the existing methods, this involving the use of a derivative mean value estimate. A second objective of this paper is the simplification of the bounds of [14, Lemma 3.2] to a convenient form of the same asymptotic strength, the present bounds being excessively unwieldy in many applications.

The investigation of the fractional parts of polynomials dates back to the start of this century, with work of Weyl [13] and then Vinogradov [12]. Heilbronn [6] refined Vinogradov's work, and later Danicic [3] obtained the natural generalisation to show that when $\alpha$ is a real number, and $k$ is a natural number exceeding 1 , then for each $\varepsilon>0$ there is a real number $N(\varepsilon, k)$ such that whenever $N \geq N(\varepsilon, k)$, we have

$$
\min _{1 \leq n \leq N}\left\|\alpha n^{k}\right\|<N^{\varepsilon-2^{1-k}} .
$$

There has since been extensive work on these and related problems by a number of authors (see, for example, $[1,2]$ ).

In this paper we shall be concerned with the situation in which $k$ is large, small values of $k$ being better dealt with elsewhere in the light of [11]. We shall require a little notation in order to describe our results. For each $s, k \in \mathbb{N}$, we define $\delta_{s, k}$ to be the unique positive solution of the equation

$$
\delta_{s, k}+\log \delta_{s, k}=1-\frac{2 s}{k} .
$$


Theorem 1.1. Define $\sigma(k)(k \geq 4)$ by

$$
\sigma(k)=\max _{s \geq 2} \frac{k\left(1-2 \delta_{s, k}\right)}{4 s^{2}-1} .
$$

Let $\alpha \in \mathbb{R}$ and $\varepsilon>0$. Then there are infinitely many $n \in \mathbb{N}$ with $\left\|\alpha n^{k}\right\| \leq$ $n^{\varepsilon-\sigma(k)}$.

A simple optimisation yields the following corollary.

COROLlary 1. The conclusion of Theorem 1.1 holds with $\sigma(k)^{-1}=$ $(\gamma+o(1)) k$, where $\gamma=9.0267256 \ldots$ Here,

$$
\gamma=\frac{(\omega+\log \omega-1)^{2}}{1-2 \omega},
$$

where $\omega=0.2818257 \ldots$ is the unique positive solution of the equation $\omega+$ $2-\omega^{-1}=\log \omega$.

This unlocalised result may be compared with [5, Theorem 2], where a bound of the above form is established with $\gamma \approx 14.4245$. By simply taking $s=k$ in Theorem 1.1 we deduce the following corollary.

COROllary 2. The conclusion of Theorem 1.1 holds with $(k \sigma(k))^{-1}=$ 9.028 .

Turning our attention now to localised bounds, we obtain the following result.

Theorem 1.2. For $k \geq 4$, define $\tau(k)$ by

$$
\tau(k)=\max _{s \geq 1} \frac{k-(k-1) \Delta_{2 s}}{4 s(2 k-1)-1-\Delta_{2 s}},
$$

where $\Delta_{s}$ is defined for each $s \in \mathbb{N}$ from (1.1) by $\Delta_{s}=k \delta_{s, k}$. Let $\alpha \in \mathbb{R}$ and $\varepsilon>0$. Then there is a real number $N(\varepsilon, k)$ such that whenever $N \geq N(\varepsilon, k)$,

$$
\min _{1 \leq n \leq N}\left\|\alpha n^{k}\right\| \leq N^{\varepsilon-\tau(k)} .
$$

COROLLARY. The conclusion of Theorem 1.2 holds with

$$
\tau(k)^{-1}=2 k(\log k+\log \log k+2+o(1)) .
$$

The latter may be compared with [1, Theorem 6.2], which gives a similar result with $\tau(k)^{-1}=4 k(\log k+\log \log k+3)$.

Our methods are based on estimates for the number, $S_{s}(P, R)$, of solutions of the equation

$$
x_{1}^{k}+\ldots+x_{s}^{k}=y_{1}^{k}+\ldots+y_{s}^{k},
$$

with $x_{i}, y_{i} \in \mathcal{A}(P, R)$, where here, and throughout,

$$
\mathcal{A}(P, R)=\{n \leq P: p \mid n, p \text { prime } \Rightarrow p \leq R\} .
$$


When $R=P^{\eta}$, with $\eta$ a sufficiently small positive number, estimates are available for $S_{s}(P, R)$ through the work of Vaughan and Wooley [9, 10, 11, 14]. In Section 2 we derive a simplification of [14, Lemma 3.2] of use in later sections, where we shall incorporate such estimates for $S_{s}(P, R)$ into the methods of R. C. Baker [1, Chapter 6], and Heath-Brown [5, Section 5]. We are able to obtain some further small improvements by considering bounds for the number, $U_{s}(P, H, R)$, of solutions of the diophantine equation

$$
h_{1} x_{1}^{k}+\ldots+h_{s} x_{s}^{k}=g_{1} y_{1}^{k}+\ldots+g_{s} y_{s}^{k},
$$

with

$$
1 \leq h_{i}, g_{i} \leq H \quad \text { and } \quad x_{i}, y_{i} \in \mathcal{A}(P, R) \quad(1 \leq i \leq s) .
$$

The estimate $U_{s}(P, H, R) \ll H^{2 s} S_{s}(P, R)$ is almost immediate on applying Hölder's inequality. In Section 3 we will demonstrate that non-trivial estimates are available in which a factor of $H$ is saved. It is these estimates which we apply in Sections 4 and 5 to deduce Theorems 1.1 and 1.2. We note here that, as experts will recognise, the methods of this paper yield the same conclusion in Theorem 1.1 with $\alpha n^{k}$ replaced by $\alpha n^{k}+\beta$, where $\beta$ is any real number, and $\alpha$ is irrational.

It transpires that the estimate established in Section 2 is of sufficient asymptotic strength to improve slightly on upper bounds in Waring's problem. Thus, denoting by $G(k)$ the smallest number $s$ such that every sufficiently large natural number is the sum of, at most, $s k$ th powers of natural numbers, in Section 6 we are able to establish the following refinement of [14, Corollary 1.2.1].

TheOrem 1.3. When $k$ is large,

$$
G(k)<k\left(\log k+\log \log k+2+\log 2+(1+o(1)) \frac{\log \log k}{\log k}\right) .
$$

For comparison, the bound given in the aforementioned corollary is $G(k) \leq k(\log k+\log \log k+O(1))$.

Much of the work for this paper was completed while the author was enjoying the hospitality of the Institute for Advanced Study, while in receipt of NSF grant DMS-8610730, and completed with the aid of a Rackham Faculty Fellowship at the University of Michigan. The author thanks the referee for useful comments.

Throughout, the implicit constant in Vinogradov's notation will depend at most on $s, k, \varepsilon$ and $\eta$, unless otherwise indicated, and $P$ denotes the basic parameter, a real number sufficiently large in terms of the latter quantities. Also, we write $[x]$ for the integer part of $x$, and $e(\alpha)$ for $e^{2 \pi i \alpha}$. 
2. A new manifestation of a mean value theorem. In this section we aim to establish a simplification of [14, Lemma 3.2], which is, moreover, asymptotically of the same strength. The following theorem provides a suitable result which is particularly useful when $s$ is $O(k)$.

Theorem 2.1. Let $k \geq 4$ and $t \in \mathbb{N}$. For each $s \in \mathbb{N}$ with $2 \leq s \leq t$, define $\delta_{s, k}$ as in (1.1) and write

$$
\lambda_{s}=2 s-k\left(1-\delta_{s, k}\right) .
$$

Then given $\varepsilon>0$, there is an $\eta_{0}=\eta_{0}(k, \varepsilon, t)$ such that whenever $0<\eta<\eta_{0}$ and $2 \leq s \leq t$,

$$
S_{s}\left(P, P^{\eta}\right) \ll P^{\lambda_{s}+\varepsilon} .
$$

Proof. We shall prove that (2.2) holds with (2.1) for each $s \geq 2$ by induction. First observe that for each $s$ the number $\delta_{s, k}$ satisfies $0<\delta_{s, k}<$ 1. Also, $\delta+\log \delta$ is an increasing function of $\delta$ when $\delta>0$. Thus the inductive hypothesis holds for $s$ provided that we can establish that

$$
S_{s}\left(P, P^{\eta}\right) \ll P^{2 s-k(1-\delta)+\varepsilon}
$$

with $\delta+\log \delta \leq \delta_{s, k}+\log \delta_{s, k}$. It therefore follows immediately from the inequality

$$
1-2 / k+\log (1-2 / k)<1-4 / k=\delta_{2, k}+\log \delta_{2, k},
$$

and the classical estimate $S_{2}\left(P, P^{\eta}\right) \ll P^{2+\varepsilon}$, that the inductive hypothesis holds with $s=2$.

Now suppose that the inductive hypothesis holds for $s$, and write $\delta=$ $\delta_{s, k}$. Let $\lambda=2 s+2-k+\Delta$, where $\Delta=k \delta(1-\theta)+k \theta-1$ and

$$
k \theta=\frac{1}{1+\delta}+\left(1-\frac{1}{1+\delta}\right)\left(\frac{1-\delta}{2}\right)^{k-1} .
$$

Then it follows from [14, Lemma 3.2] that whenever $0<\eta<\eta_{1}(k, \varepsilon, s)$, we have $S_{s+1}\left(P, P^{\eta}\right) \ll P^{\lambda+\varepsilon}$. Therefore, if we can prove that

$$
\frac{\Delta}{k}+\log \frac{\Delta}{k} \leq \delta_{s+1, k}+\log \delta_{s+1, k},
$$

then we may conclude from the above remarks that the inductive hypothesis follows with $s$ replaced by $s+1$.

From (2.3) we obtain

$$
k \theta \leq \frac{1}{1+\delta}\left(1+\delta 2^{1-k}\right),
$$

and hence

$$
\Delta=k \delta+k(1-\delta) \theta-1 \leq k \delta+\frac{1-\delta}{1+\delta}\left(1+\delta 2^{1-k}\right)-1 .
$$


On writing $\omega=(1-\delta) 2^{1-k}$, we therefore deduce that

$$
\Delta \leq k \delta\left(1-\frac{2-\omega}{k(1+\delta)}\right),
$$

and hence

$$
\frac{\Delta}{k}+\log \frac{\Delta}{k} \leq \delta\left(1-\frac{2-\omega}{k(1+\delta)}\right)+\log \delta+\log \left(1-\frac{2-\omega}{k(1+\delta)}\right) .
$$

But a simple estimate for $\log x$ gives

$$
\log \left(1-\frac{2-\omega}{k(1+\delta)}\right) \leq-\frac{2-\omega}{k(1+\delta)}-\frac{(2-\omega)^{2}}{2 k^{2}(1+\delta)^{2}} .
$$

Then on writing

we obtain

$$
E=2 k \omega-\frac{(2-\omega)^{2}}{(1+\delta)^{2}}
$$

$$
\frac{\Delta}{k}+\log \frac{\Delta}{k} \leq \delta+\log \delta-\frac{2}{k}+\frac{E}{2 k^{2}} .
$$

Now we observe that $2-\omega=2-(1-\delta) 2^{1-k} \geq 2-(1-\delta)=1+\delta$, and $\omega \leq 2^{1-k}$. Thus when $k \geq 4$, we have $E \leq k 2^{2-k}-1 \leq 0$, and hence by the inductive hypothesis,

$$
\frac{\Delta}{k}+\log \frac{\Delta}{k} \leq \delta_{s, k}+\log \delta_{s, k}-\frac{2}{k}=1-\frac{2 s+2}{k}=\delta_{s+1, k}+\log \delta_{s+1, k} .
$$

Then the inductive hypothesis holds with $s$ replaced by $s+1$, and the proof of the theorem is complete.

When $s$ is rather larger than $k$, it is usually more convenient to use the exponentiated version of Theorem 2.1 embodied in the following corollary.

Corollary. Let $k \geq 4$ and $t \in \mathbb{N}$. For each $s \in \mathbb{N}$ with $2 \leq s \leq t$, define the real number $\Delta_{s}$ to be the unique positive solution of the equation

$$
\Delta_{s} e^{\Delta_{s} / k}=k e^{1-2 s / k} .
$$

Then (2.2) holds with $\lambda_{s}=2 s-k+\Delta_{s}$. Consequently, (2.2) holds with

$$
\lambda_{s}=2 s-k+k e^{1-2 s / k} .
$$

Proof. The first assertion follows immediately on exponentiating (1.1) and writing $\Delta_{s}=k \delta_{s, k}$. The second assertion follows on noting that $\Delta_{s} \geq 0$ for each $s$, so that $\Delta_{s} \leq k e^{1-2 s / k}$.

3. A mean value theorem. We now set about obtaining estimates for the mean value $U_{s}(P, H, R)$ defined by (1.2) and (1.3), and also the mean 
value $V_{s}(P, H, R)$, which we define to be the number of solutions of the diophantine equation

$$
\sum_{i=1}^{s} h_{i}\left(x_{i}^{k}-y_{i}^{k}\right)=0
$$

with $h_{i}, x_{i}, y_{i}$ satisfying (1.3). We start with a very simple estimate, useful when $s$ is even.

Lemma 3.1. When $r \in \mathbb{N}$ we have

$$
U_{2 r}(P, H, R) \ll P^{\varepsilon} H^{4 r-1+\varepsilon} S_{2 r}(P, R)+H^{4 r}\left(S_{r}(P, R)\right)^{2}
$$

and

$$
V_{2 r}(P, H, R) \ll P^{\varepsilon} H^{2 r-1+\varepsilon} S_{2 r}(P, R)+H^{2 r}\left(S_{r}(P, R)\right)^{2} .
$$

Further, for each $s \in \mathbb{N}$ we have the trivial estimates

$$
U_{s}(P, H, R) \ll H^{2 s} S_{s}(P, R) \quad \text { and } \quad V_{s}(P, H, R) \ll H^{s} S_{s}(P, R) .
$$

Proof. Let

$$
f(\alpha)=\sum_{x \in \mathcal{A}(P, R)} e\left(\alpha x^{k}\right) .
$$

Then by considering the underlying diophantine equation, for each $s \in \mathbb{N}$ we have

$$
U_{s}(P, H, R)=\int_{0}^{1}\left|\sum_{1 \leq h \leq H} f(\alpha h)\right|^{2 s} d \alpha .
$$

Therefore, by Hölder's inequality,

$$
U_{s}(P, H, R) \ll H^{2 s-1} \sum_{1 \leq h \leq H} \int_{0}^{1}|f(\alpha h)|^{2 s} d \alpha=H^{2 s} S_{s}(P, R),
$$

by considering the underlying diophantine equation. A similar argument bounds $V_{s}(P, H, R)$ in an analogous manner, and thus the final line of the lemma holds.

We may therefore suppose that $s$ is even, say $s=2 r$. We now apply Cauchy's inequality to (3.1) to obtain, on considering the underlying diophantine equation,

$$
U_{s}(P, H, R) \ll H^{s} \int_{0}^{1}\left(\sum_{h \leq H}|f(\alpha h)|^{2}\right)^{s} d \alpha \ll H^{s} V_{s}(P, H, R) .
$$

Thus it suffices to consider $V_{s}(P, H, R)$. By Hölder's inequality, we have

$$
V_{2 r}(P, H, R) \ll H^{2 r-2} \int_{0}^{1}\left(\sum_{h \leq H}|f(\alpha h)|^{2 r}\right)^{2} d \alpha .
$$


The latter integral is the number of solutions of the diophantine equation

$$
h \sum_{i=1}^{r}\left(x_{i}^{k}-y_{i}^{k}\right)=g \sum_{i=1}^{r}\left(u_{i}^{k}-v_{i}^{k}\right)
$$

with $1 \leq h, g \leq H$ and $x_{i}, y_{i}, u_{i}, v_{i} \in \mathcal{A}(P, R)(1 \leq i \leq r)$. Let $R(n ; h)$ denote the number of solutions of the equation

$$
h \sum_{i=1}^{r}\left(x_{i}^{k}-y_{i}^{k}\right)=n
$$

with $x_{i}, y_{i} \in \mathcal{A}(P, R)(1 \leq i \leq r)$. Then the number of solutions of (3.2) is given by

$$
\begin{aligned}
\sum_{n}\left(\sum_{h=1}^{H} R(n ; h)\right)^{2} & =\sum_{n \neq 0}\left(\sum_{h \mid n} R(n ; h)\right)^{2}+\left(\sum_{h=1}^{H} R(0 ; h)\right)^{2} \\
& \ll(P H)^{\varepsilon} \sum_{n \neq 0} \sum_{h=1}^{H} R(n ; h)^{2}+\left(H S_{r}(P, R)\right)^{2},
\end{aligned}
$$

by Cauchy's inequality and a standard estimate for the divisor function. Then by considering the underlying diophantine equation,

$$
V_{2 r}(P, H, R) \ll P^{\varepsilon} H^{2 r-1+\varepsilon} S_{2 r}(P, R)+H^{2 r}\left(S_{r}(P, R)\right)^{2} .
$$

The lemma now follows immediately.

We shall now briefly outline how the new iterative method in Waring's problem may be applied successfully to provide non-trivial estimates for the mean value $U_{s}(P, H, R)$ under more general circumstances. We begin with an estimate for a fourth moment.

Lemma 3.2. We have $U_{2}(P, H, R) \ll H^{3+\varepsilon} P^{2+\varepsilon}$.

Pro of. Write

$$
F(\alpha)=\sum_{1 \leq x \leq P} e\left(\alpha x^{k}\right)
$$

Then by considering the underlying diophantine equation, we have

$$
\begin{aligned}
U_{2}(P, H, R) & \leq \int_{0}^{1}\left|\sum_{1 \leq h \leq H} F(\alpha h)\right|^{4} d \alpha \\
& \leq H \int_{0}^{1} \sum_{1 \leq g \leq H}|F(\alpha g)|^{2}\left|\sum_{1 \leq h \leq H} F(\alpha h)\right|^{2} d \alpha,
\end{aligned}
$$

by Cauchy's inequality. The latter integral is $O(T(P, H))$, where $T(P, H)$ denotes the number of solutions of the diophantine equation

$$
g\left(x^{k}-y^{k}\right)=h_{1} u_{1}^{k}-h_{2} u_{2}^{k},
$$


with $1 \leq g, h_{1}, h_{2} \leq H$ and $1 \leq x, y, u_{1}, u_{2} \leq P$. The number of solutions of (3.3) counted by $T(P, H)$ in which $x=y$ is $O\left((H P)^{2+\varepsilon}\right)$. Meanwhile, if $x \neq y$, we may assign $h_{1}, h_{2}, u_{1}, u_{2}$ in at most $H^{2} P^{2}$ ways, and then $g, x-y$ and $x^{k-1}+x^{k-2} y+\ldots+y^{k-1}$ are divisors of a fixed non-zero integer. Thus, by using standard estimates for the divisor function, the number of solutions in this case is $O\left(\left(H^{2} P^{2}\right)^{1+\varepsilon}\right)$. This completes the proof of the lemma.

We now set about constructing an iterative method for $U_{s}(P, H, R)$, in a manner entirely analogous to the method of [14]. Let $\Psi(z, \mathbf{c})$ denote a polynomial with integer coefficients in the variables $z, c_{1}, \ldots, c_{t}$ of degree in $z$ at least one, and write $\Psi^{\prime}(z, \mathbf{c})$ for $(\partial \Psi / \partial z)(z, \mathbf{c})$. Let $H, P, Q$, and $R$ be positive real numbers with $R \leq Q \leq P$, and let $C_{i}, C_{i}^{\prime}(1 \leq i \leq t)$ be real numbers with $1 \leq C_{i}^{\prime} \leq C_{i} \ll P$. Let

$$
R_{s}(P, Q, H, R)=R_{s}\left(P, Q, H, R ; \Psi ; \mathbf{C}, \mathbf{C}^{\prime}\right)
$$

denote the number of solutions of the equation

$$
h \Psi(z, \mathbf{c})+h_{1} x_{1}^{k}+\ldots+h_{s} x_{s}^{k}=g \Psi\left(z^{\prime}, \mathbf{c}^{\prime}\right)+g_{1} y_{1}^{k}+\ldots+g_{s} y_{s}^{k}
$$

with

$$
\begin{gathered}
x_{j}, y_{j} \in \mathcal{A}(Q, R) \quad \text { and } \quad 1 \leq h, g, h_{j}, g_{j} \leq H \quad(1 \leq j \leq s), \\
1 \leq z, z^{\prime} \leq P, \quad C_{i}^{\prime}<c_{i}, c_{i}^{\prime} \leq C_{i} \quad(1 \leq i \leq t) .
\end{gathered}
$$

For a given real number $\theta$ with $1<P^{\theta}<Q$, let

$$
W_{s}(P, Q, H, R ; \theta)=W_{s}\left(P, Q, H, R ; \theta ; \Psi ; \mathbf{C}, \mathbf{C}^{\prime}\right)
$$

denote the number of solutions of the equation

$$
h\left(\Psi(z, \mathbf{c})-\Psi\left(z^{\prime}, \mathbf{c}\right)\right)+w^{k} \sum_{i=1}^{s}\left(h_{i} u_{i}^{k}-g_{i} v_{i}^{k}\right)=0
$$

with $z, z^{\prime}, \mathbf{c}, h, h_{i}, g_{i}$ as in (3.5) and (3.6), and

$$
\begin{gathered}
P^{\theta}<w \leq \min \left\{Q, P^{\theta} R\right\}, \quad u_{j}, v_{j} \in \mathcal{A}\left(Q P^{-\theta}, R\right) \quad(1 \leq j \leq s), \\
z \equiv z^{\prime}\left(\bmod w^{k}\right) .
\end{gathered}
$$

Finally, let $N_{s}(P, Q, H, R)=N_{s}\left(P, Q, H, R ; \Psi ; \mathbf{C}, \mathbf{C}^{\prime}\right)$ denote the number of solutions of the equation (3.4) subject to (3.5), (3.6) and also $\Psi^{\prime}(z, \mathbf{c})=$ $\Psi^{\prime}\left(z^{\prime}, \mathbf{c}^{\prime}\right)=0$.

The lemma below relates $R_{s}$ to $W_{s}$.

Lemma 3.3. Let $\theta=\theta(s, k ; \Psi)$ satisfy $1<P^{\theta}<Q$. Then

$$
\begin{aligned}
R_{s}(P, Q, H, R) \ll & R_{s}\left(P, P^{\theta}, H, R\right)+N_{s}(P, Q, H, R) \\
& +H^{2} Q P^{\theta+\varepsilon} R_{s-1}(P, Q, H, R) \\
& +H P^{\varepsilon}\left(\prod_{i=1}^{t} C_{i}\right)\left(P^{\theta} R\right)^{2 s-1} W_{s}(P, Q, H, R ; \theta) .
\end{aligned}
$$


The implied constant may depend on $\Psi$.

Proof. The proof follows that of [14, Lemma 2.2], with obvious modifications. The condition (2.12) of that proof should be replaced by

$$
x_{j} \mathcal{D}\left(P^{\theta}\right) h \Psi^{\prime}(z, \mathbf{c}) \quad \text { or } \quad y_{j} \mathcal{D}\left(P^{\theta}\right) g \Psi^{\prime}\left(z^{\prime}, \mathbf{c}^{\prime}\right),
$$

with similar changes for each occurrence of $\Psi^{\prime}$.

Now follow the same change of notation, with obvious modifications, as that following [14, Lemma 2.2]. We record two further lemmata.

Lemma 3.4. Suppose that for some positive real number $\eta$,

$$
\exp \left((\log \log P)^{2}\right)<R \leq P^{\eta} .
$$

Then for $j=0, \ldots, k-1$, and for any constant $\kappa>0$,

$$
\begin{aligned}
& R_{s}\left(P, Q_{j}, H, R ; \Psi_{j} ; \kappa\right) \\
& \quad \ll H P^{\varepsilon}\left(\prod_{i=1}^{j} H_{i} M_{i} R\right)\left(M_{j+1} R\right)^{2 s-1} W_{s}\left(P, Q_{j}, H, R ; \phi_{j+1} ; \Psi_{j} ; \kappa\right) .
\end{aligned}
$$

(Here the convention that $Q_{0}=P$ is adopted.)

Proof. This follows precisely as in the proof of [14, Lemma 2.3], with obvious modifications.

Lemma 3.5. For $j=0,1, \ldots, k-1$, and any constant $\kappa \geq 1$,

$$
\begin{aligned}
W_{s}\left(P, Q_{j}, H, R ; \phi_{j+1} ; \Psi_{j} ; \kappa\right) & \\
\ll & P H M_{j+1} R\left(\prod_{i=1}^{j} H_{i} M_{i} R\right) U_{s}\left(Q_{j+1}, H, R\right) \\
& \quad+\left(U_{s}\left(2 Q_{j+1}, H, R\right) R_{s}\left(2 P, 2 Q_{j+1}, H, R ; \Psi_{j+1} ; 2 \kappa\right)\right)^{1 / 2} .
\end{aligned}
$$

Proof. This follows the proof of [14, Lemma 3.1].

We may now follow the argument of the proof of [14, Lemma 3.2] to obtain the following lemma.

Lemma 3.6. Suppose that $t$ is a positive integer and $\mu$ is a positive real number with

$$
2 t-k<\mu \leq 2 t
$$

and satisfying the property that, given $\varepsilon>0$, there is a positive number $\eta_{0}=$ $\eta_{0}(k, \varepsilon)$ such that whenever $0<\eta<\eta_{0}$, then $U_{t}\left(P, H, P^{\eta}\right) \ll H^{2 t-1+\varepsilon} P^{\mu+\varepsilon}$. 
Define the real numbers $\lambda_{s}, \theta_{s}, \Delta_{s}(s=t, t+1, \ldots)$ successively by $\lambda_{t}=\mu, \theta_{t}=0, \Delta_{t}=\mu-2 t+k$, and for $s>t, b y$

$$
\begin{aligned}
& \theta_{s}=\frac{1}{k+\Delta_{s-1}}+\left(\frac{1}{k}-\frac{1}{k+\Delta_{s-1}}\right)\left(\frac{k-\Delta_{s-1}}{2 k}\right)^{k-1}, \\
& \Delta_{s}=\Delta_{s-1}\left(1-\theta_{s}\right)+k \theta_{s}-1, \quad \lambda_{s}=2 s-k+\Delta_{s} .
\end{aligned}
$$

Then given $t^{\prime}$ and $\varepsilon^{\prime}>0$, there is an $\eta_{1}=\eta_{1}\left(k, \varepsilon^{\prime}, t^{\prime}\right)$ such that whenever $0<\eta<\eta_{1}$ and $t \leq s \leq t^{\prime}$,

$$
U_{s}\left(P, H, P^{\eta}\right) \ll H^{2 s-1+\varepsilon} P^{\lambda_{s}+\varepsilon^{\prime}} .
$$

We may now apply Lemma 3.6 with $t=2$, and make use of Lemma 3.2. Thus, by the argument of the proof of Theorem 2.1 we may finally conclude with the following estimate.

LEMma 3.7. Let $\lambda_{s}$ be defined as in the statement of the corollary to Theorem 2.1. Then under the same hypotheses as in the statement of Theorem 2.1, for each $k \geq 4$ and $s \geq 2$ we have

$$
U_{s}\left(P, H, P^{\eta}\right) \ll H^{2 s-1+\varepsilon} P^{\lambda_{s}+\varepsilon} .
$$

We note that the preceding argument is really intended as a demonstration that non-trivial estimates for $U_{s}$ can be obtained. The methods of Sections 4 and 5 are most effective when $k$ is small, and under such circumstances the estimates of [11] supersede those of Theorem 2.1. Nonetheless, the argument of this section provides a basis for the estimation of $U_{s}(P, H, R)$ through the methods of [11].

4. Unlocalised bounds for fractional parts of $\alpha n^{k}$. We are now in a position to obtain unlocalised bounds for the fractional parts of $\alpha n^{k}$ by using estimates for $U_{s}(P, H, R)$ arising from Lemma 3.7 and Theorem 2.1. This we achieve by refining an argument of Heath-Brown [5, Section 5]. We shall require the following lemma due to Harman.

Lemma 4.1. Suppose that $g(n)$ is a non-negative function for all $n, L$ is a positive integer and $\alpha_{n}$ is a sequence of real numbers for $n=1, \ldots, N$. If

$$
\sum_{l=1}^{L}\left|\sum_{n=1}^{N} g(n) e\left(l \alpha_{n}\right)\right|<\frac{1}{6} \sum_{n=1}^{N} g(n),
$$

then for any $\beta$, there is a solution of $\left\|\alpha_{n}+\beta\right\|<L^{-1}$, with $1 \leq n \leq N$.

Proof. This is Harman [4, Lemma 5].

Given positive numbers $P, M$ and $R$, we define the arithmetical function $g(n)=g(n ; P, M, R)$ by

$$
g(n)=\operatorname{card}\left\{(x, y) \in \mathbb{N}^{2}: x y=n, x \in \mathcal{A}(P, R), y \in \mathcal{A}(M, R)\right\} .
$$


Thus, in particular, $g(n)$ is zero when $n \notin \mathcal{A}(P, R)$, and for all $n \in \mathbb{N}$ we have $g(n) \leq d(n) \ll_{\varepsilon} n^{\varepsilon}$. We now describe the exponential sum at the core of our argument. When $N$ and $H$ are large positive numbers, we define the positive numbers $P$ and $M$ by $P^{2}=N H^{-1 / k}$ and $M^{2}=N H^{1 / k}$, and when $\alpha \in \mathbb{R}$ we write

$$
S(\alpha ; H, N, R)=\sum_{h=1}^{H}\left|\sum_{n=1}^{N} g(n ; P, M, R) e\left(\alpha h n^{k}\right)\right| .
$$

We now provide a result which bounds the exponential sum $S(\alpha ; H, N, R)$.

Lemma 4.2. Suppose that $s \in \mathbb{N}$, and that $\delta_{s, k}$ is defined as in (1.1). Let $\alpha \in \mathbb{R}$, and suppose that $a \in \mathbb{Z}$ and $q \in \mathbb{N}$ satisfy $(a, q)=1$ and $|q \alpha-a| \leq q^{-1}$. Then when $R \leq N^{\eta}$ with $\eta$ a sufficiently small positive number (in the context of Theorem 2.1),

$$
S(\alpha ; H, N, R)^{2 s^{2}} \ll_{\varepsilon} q^{\varepsilon}(H N)^{2 s^{2}+\varepsilon} N^{k \delta_{s, k}}\left(q^{-1}+\left(H N^{k}\right)^{-1 / 2}+q H^{-1} N^{-k}\right) .
$$

Proof. For the sake of conciseness, we write $S(\alpha)$ for $S(\alpha ; H, N, R)$. From the definition of $g(n)$, it follows that

$$
S(\alpha)=\sum_{1 \leq h \leq H}\left|\sum_{x \in \mathcal{A}(P, R)} \sum_{y \in \mathcal{A}(M, R)} e\left(\alpha h(x y)^{k}\right)\right| .
$$

Define the complex numbers of unit modulus, $\varepsilon_{x, h}$, by

$$
\left|\sum_{y \in \mathcal{A}(M, R)} e\left(\alpha h(x y)^{k}\right)\right|^{s}=\varepsilon_{x, h}\left(\sum_{y \in \mathcal{A}(M, R)} e\left(\alpha h(x y)^{k}\right)\right)^{s} .
$$

Also, let $r_{c}$ denote the number of solutions of the diophantine equation

$$
y_{1}^{k}+\ldots+y_{s}^{k}=c
$$

with $y_{i} \in \mathcal{A}(M, R)(1 \leq i \leq s)$. Then by applying Hölder's inequality to (4.1), we obtain

$$
\begin{aligned}
|S(\alpha)|^{s} & \leq(P H)^{s-1} \sum_{1 \leq h \leq H} \sum_{x \in \mathcal{A}(P, R)}\left|\sum_{y \in \mathcal{A}(M, R)} e\left(\alpha h(x y)^{k}\right)\right|^{s} \\
& =(P H)^{s-1} \sum_{c} r_{c} \sum_{h} \sum_{x} \varepsilon_{x, h} e\left(\alpha c h x^{k}\right) .
\end{aligned}
$$

A second application of Hölder's inequality yields

$$
|S(\alpha)|^{2 s^{2}} \leq(P H)^{2 s(s-1)} J_{s}(\alpha)\left(\sum_{c} r_{c}\right)^{2 s-2}\left(\sum_{c} r_{c}^{2}\right),
$$

where

$$
J_{s}(\alpha)=\sum_{1 \leq c \leq s M^{k}}\left|\sum_{h} \sum_{x} \varepsilon_{x, h} e\left(\alpha \operatorname{ch} x^{k}\right)\right|^{2 s} .
$$


But by considering the underlying diophantine equations, we have $\sum_{c} r_{c} \leq$ $M^{s}$ and $\sum_{c} r_{c}^{2}=S_{s}(M, R)$, and hence

$$
|S(\alpha)|^{2 s^{2}} \leq(P H M)^{2 s(s-1)} J_{s}(\alpha) S_{s}(M, R) .
$$

Let $\widetilde{r}_{d}$ denote the number of solutions of the equation

$$
\sum_{i=1}^{s} h_{i} x_{i}^{k}-\sum_{i=s+1}^{2 s} h_{i} x_{i}^{k}=d,
$$

with $x_{i} \in \mathcal{A}(P, R)$ and $1 \leq h_{i} \leq H(1 \leq i \leq 2 s)$, each solution weighted by the factor

$$
\left(\prod_{i=1}^{s} \varepsilon_{x_{i}, h_{i}}\right)\left(\prod_{i=s+1}^{2 s} \bar{\varepsilon}_{x_{i}, h_{i}}\right) .
$$

Then by considering the underlying diophantine equation,

$$
\widetilde{r}_{d}=\int_{0}^{1}\left|\sum_{x} \sum_{h} \varepsilon_{x, h} e\left(\gamma x^{k} h\right)\right|^{2 s} e(-\gamma d) d \gamma .
$$

Therefore, on making a trivial estimate, $\left|\widetilde{r}_{d}\right| \leq \widetilde{r}_{0} \leq U_{s}(P, H, R)$, since $\left|\varepsilon_{x, h}\right|=1$. On recalling equation (4.2), we have

$$
\begin{aligned}
& J_{s}(\alpha) \\
& =\sum_{1 \leq c \leq s M^{k}} \sum_{d} \widetilde{r}_{d} e(\alpha c d) \leq U_{s}(P, H, R) \sum_{|d| \leq s H P^{k}}\left|\sum_{1 \leq c \leq s M^{k}} e(\alpha c d)\right| .
\end{aligned}
$$

But, by using [8, Lemma 2.2] we obtain

$$
\begin{aligned}
\sum_{|d| \leq s H P^{k}} \mid & \sum_{1 \leq c \leq s M^{k}} e(\alpha c d) \mid \\
& \leq \sum_{|d| \leq s H P^{k}} \min \left\{s M^{k},\|\alpha d\|^{-1}\right\} \\
& \ll M^{k}+(q H P)^{\varepsilon}(P M)^{k} H\left(q^{-1}+M^{-k}+q H^{-1}(P M)^{-k}\right) .
\end{aligned}
$$

On recalling the definitions of $P$ and $M$, we have $P^{k} H=M^{k}$, and hence by (4.3), (4.4), Lemma 3.7 and Theorem 2.1,

$S(\alpha)^{2 s^{2}}$

$\ll q^{\varepsilon}(N H)^{2 s(s-1)+2 \varepsilon} H^{2 s-1}(P M)^{2 s-k+k \delta_{s, k}} N^{k} H\left(q^{-1}+M^{-k}+q H^{-1} N^{-k}\right)$.

Finally, we deduce that

$$
S(\alpha)^{2 s^{2}} \ll q^{\varepsilon}(N H)^{2 s^{2}+2 \varepsilon} N^{k \delta_{s, k}}\left(q^{-1}+M^{-k}+q H^{-1} N^{-k}\right) .
$$

This completes the proof of the lemma. 
We are now in a position to prove Theorem 1.1 in a standard manner. First note that the theorem is trivial if $\alpha$ is rational, so we suppose that $\alpha$ is irrational. Take $a / q$ to be a convergent to the continued fraction of $\alpha$, so that $(a, q)=1$ and $|q \alpha-a| \leq q^{-1}$. We take $\sigma(k)$ to be as in the statement of Theorem 1.1, with $s$ the value of the parameter corresponding to the maximum. Let $\phi$ be any real number with $\varepsilon<\phi<\sigma(k)$, and define the real numbers $N$ and $H$ by

$$
N^{k+\sigma(k)-\phi}=q^{2} \quad \text { and } \quad H=N^{\sigma(k)-\phi} .
$$

Then by Lemma 4.2, we have

$$
S(\alpha ; H, N, R)^{2 s^{2}} \ll_{\varepsilon}(H N)^{2 s^{2}} N^{k \delta_{s, k}+\varepsilon-k / 2} H^{-1 / 2} .
$$

But by using the definitions of $\sigma(k)$ and $H$, we discover that

$$
N^{k \delta_{s, k}+\varepsilon-k / 2} H^{-1 / 2} \ll N^{\psi},
$$

where

$$
2 \psi=2 \varepsilon-k\left(1-2 \delta_{s, k}\right)-\frac{k\left(1-2 \delta_{s, k}\right)}{4 s^{2}-1}+\phi=2 \varepsilon+\phi-4 s^{2} \sigma(k) .
$$

Thus $S(\alpha ; H, N, R)=o(N)$. But as a simple deduction from, for example, [9, Lemma 5.3], we have $\sum_{n=1}^{N} g(n) \gg N$, and hence $S(\alpha ; H, N, R)=$ $o\left(\sum_{n=1}^{N} g(n)\right)$. Therefore, by Lemma 4.1 with $L=H$, it follows that

$$
\min _{1 \leq n \leq N}\left\|\alpha n^{k}\right\|<H^{-1}=N^{\phi-\sigma(k)},
$$

and Theorem 1.1 follows on taking a sequence of $q$ going to infinity.

The deduction of the corollaries to Theorem 1.1 may now be disposed of swiftly. For Corollary 1 we take $s=[C k]+1$, with $C$ a positive number to be chosen later. Then in Theorem 1.1 we have $k \sigma(k)=\zeta+o(1)$, where

$$
4 C^{2} \zeta=1-2 \delta,
$$

and by equation (1.1),

$$
\delta+\log \delta=1-2 C .
$$

On eliminating $C$, we find that the optimal choice of parameters is determined by the equation

$$
\delta+2-\delta^{-1}=\log \delta .
$$

Thus we obtain $C=0.99232 \ldots, \delta=0.28182 \ldots$, and $\zeta^{-1}=9.02672 \ldots$ Also, on taking $s=k$ in Theorem 1.1 we obtain $4 k \sigma(k) \geq 1-2 \delta$, where by equation (1.1), $\delta+\log \delta=-1$. Corollary 2 follows with a little calculation.

5. Localised bounds. We now turn our attention to the matter of obtaining localised bounds for the fractional part of $\alpha n^{k}$ through the estimates 
of Sections 2 and 3. Once again we shall make use of Lemma 4.1, this time through the vehicle of a variant of Vinogradov's estimate for exponential sums based on [7, Section 2].

Given positive numbers $P, M$ and $R$, we define the arithmetical function $g(n)=g(n ; P, M, R)$ by

$$
g(n)=\operatorname{card}\left\{(p, x) \in \mathbb{N}^{2}: p x=n, p \text { prime, } P<p \leq 2 P, x \in \mathcal{A}(M, R)\right\} .
$$

Thus, in particular, for all $n \in \mathbb{N}$ we have $g(n) \leq d(n) \ll_{\varepsilon} n^{\varepsilon}$. We now describe the exponential sum at the core of our argument. When $N$ and $H$ are large positive numbers, we define the positive numbers $P$ and $M$ by $(2 P)^{2 k-1}=N^{k} H$ and $M^{2 k-1}=N^{k-1} H^{-1}$, and when $\alpha \in \mathbb{R}$ we write

$$
T(\alpha ; H, N, R)=\sum_{h=1}^{H}\left|\sum_{n=1}^{N} g(n ; P, M, R) e\left(\alpha h n^{k}\right)\right| .
$$

Lemma 5.1. Suppose that $s \in \mathbb{N}$, and that $\Delta_{s}$ and $\Delta_{2 s}$ are defined as in the corollary to Theorem 2.1. Let $\alpha \in \mathbb{R}$, and suppose that $a \in \mathbb{Z}$ and $q \in \mathbb{N}$ satisfy $(a, q)=1, q \leq 2(2 P)^{k}$ and $|q \alpha-a| \leq \frac{1}{2}(2 P)^{-k}$, and suppose further that if $q \leq P$, then $|q \alpha-a| \gg P H^{-1} N^{-k}$. Then when $R \leq N^{\eta}$, with $\eta$ a sufficiently small positive number (in the context of Theorem 2.1),

$$
T(\alpha ; H, N, R)^{4 s} \ll_{\varepsilon}(H N)^{4 s+\varepsilon}\left(P^{-1} M^{\Delta_{2 s}}+P^{-1} H M^{2 \Delta_{s}-k}\right) .
$$

Proof. For the sake of conciseness, we write $T(\alpha)$ for $T(\alpha ; H, N, R)$. From the definition of $g(n)$, it follows that

$$
T(\alpha)=\sum_{1 \leq h \leq H}\left|\sum_{P<p \leq 2 P} \sum_{x \in \mathcal{A}(M, R)} e\left(\alpha h(p x)^{k}\right)\right|,
$$

where the summation over $p$ is over prime numbers. Let $b_{y}$ denote the number of solutions of the diophantine equation

$$
h_{1}\left(x_{1}^{k}-y_{1}^{k}\right)+\ldots+h_{s}\left(x_{s}^{k}-y_{s}^{k}\right)=y,
$$

with $1 \leq h_{i} \leq H$ and $x_{i}, y_{i} \in \mathcal{A}(M, R)(1 \leq i \leq s)$. We note that $b_{y}=b_{-y}$. Also, write $Y=s H M^{k}$. Then by applying Hölder's inequality to (5.1), we obtain

$$
\begin{aligned}
|T(\alpha)|^{2 s} & \ll H^{s} P^{2 s-1} \sum_{P<p \leq 2 P}\left(\sum_{1 \leq h \leq H}\left|\sum_{x \in \mathcal{A}(M, R)} e\left(\alpha h(p x)^{k}\right)\right|^{2}\right)^{s} \\
& =H^{s} P^{2 s-1} \sum_{P<p \leq 2 P} \sum_{|y| \leq Y} b_{y} e\left(\alpha p^{k} y\right) .
\end{aligned}
$$


But by the lemma of [7, Section 2], it follows that

$$
\begin{aligned}
\sum_{P<p \leq 2 P} \sum_{|y| \leq Y} b_{y} e\left(\alpha p^{k} y\right) & \ll \sum_{P<p \leq 2 P}\left(b_{0}+2 \operatorname{Re} \sum_{1 \leq y \leq Y} b_{y} e\left(\alpha p^{k} y\right)\right) \\
& \ll P^{\varepsilon}\left(P Y+P^{k}\right)^{1 / 2}\left(\sum_{|y| \leq Y} b_{y}^{2}\right)^{1 / 2} .
\end{aligned}
$$

We now note that $P H M^{k} \asymp P^{k}$, and hence from (5.2),

$$
|T(\alpha)|^{4 s} \ll P^{2 \varepsilon} H^{2 s+1} P^{4 s-1} M^{k} V_{2 s}(M, H, R) .
$$

Thus, by Lemma 3.1 and the corollary to Theorem 2.1, we have

$$
|T(\alpha)|^{4 s} \ll(P M H)^{4 s+2 \varepsilon}\left(P^{-1} M^{\Delta_{2 s}}+P^{-1} H M^{2 \Delta_{s}-k}\right),
$$

and the lemma now follows immediately.

We may now prove Theorem 1.2 in a standard manner. We take $\tau(k)$ to be as in the statement of Theorem 1.2, with $s$ the value of the parameter corresponding to the maximum. We take $\phi$ to be any real number with $\varepsilon<\phi<\tau(k)$. Let $N$ be a large positive number, and put $H=N^{\tau(k)-\phi}$. By Dirichlet's Theorem, we may choose $a \in \mathbb{Z}$ and $q \in \mathbb{N}$ with $(a, q)=1$, $q \leq 2(2 P)^{k}$ and $|q \alpha-a| \leq \frac{1}{2}(2 P)^{-k}$. Suppose first that when $q \leq P$, we have $|q \alpha-a| \geq P H^{-1} N^{-k}$. Then by Lemma 5.1 we have

$$
T(\alpha)^{4 s} \ll(H N)^{4 s+\varepsilon}\left(P^{-1} M^{\Delta_{2 s}}+P^{-1} H M^{2 \Delta_{s}-k}\right) .
$$

In view of the definition of $\Delta_{s}$, it follows with some calculation that when $k \geq 4$, the second term in parentheses is always smaller than the first. Then on observing that

$$
(H N)^{\varepsilon} P^{-1} M^{\Delta_{2 s}} \ll N^{\psi},
$$

where

$$
\begin{aligned}
(2 k-1) \psi & \leq 2(2 k-1) \varepsilon-k+(k-1) \Delta_{2 s}-\left(1+\Delta_{2 s}\right)(\tau(k)-\phi) \\
& <-4 s(2 k-1)(\tau(k)-\phi),
\end{aligned}
$$

we deduce that $T(\alpha)=o(N / \log N)$, and hence, $T(\alpha)=o\left(\sum_{n=1}^{N} g(n)\right)$. Then in this case we deduce from Lemma 4.1, with $L=H$, that

$$
\min _{1 \leq n \leq N}\left\|\alpha n^{k}\right\|<H^{-1}=N^{\phi-\tau(k)} .
$$

Then we may suppose that $q \leq P$ and $|q \alpha-a|<P H^{-1} N^{-k}$. Then

$$
\left\|\alpha q^{k}\right\| \leq\left|q^{k} \alpha-q^{k-1} a\right|<H^{-1} P^{k} N^{-k} \leq N^{\phi-\tau(k)} .
$$

Thus

$$
\min _{1 \leq n \leq N}\left\|\alpha n^{k}\right\| \leq\left\|\alpha q^{k}\right\|<N^{\phi-\tau(k)},
$$

and this completes the proof of Theorem 1.2. 
To prove the corollary to Theorem 1.2, we merely apply the corollary to Theorem 2.1. Putting $s=\left[\frac{1}{4} k(\log k+\log \log k+1)\right]+1$, we obtain

$$
\Delta_{2 s} \leq k e^{1-4 s / k} \leq \frac{1}{\log k} .
$$

Thus

$$
\begin{aligned}
\tau(k) & \geq \frac{k-(k-1) /(\log k)}{2 k^{2}(\log k+\log \log k+1+o(1))} \\
& =(2 k(\log k+\log \log k+2+o(1)))^{-1} .
\end{aligned}
$$

6. The upper bound for $G(k)$. The proof of Theorem 1.3 is entirely straightforward, given the corollary to Theorem 2.1. We put

$$
\mathcal{C}=\left\{x=p z: p \text { prime, } X / 2<p \leq X, z \in \mathcal{A}\left(X, X^{\eta}\right)\right\},
$$

where $X=P^{1 / 2}$, and write

$$
h(\alpha)=\sum_{x \in \mathcal{C}} e\left(\alpha x^{k}\right) .
$$

Let $\mathfrak{m}$ be the set of $\alpha \in[0,1]$ such that whenever $a \in \mathbb{Z}, q \in \mathbb{N},(a, q)=1$ and $|q \alpha-a| \leq P^{1 / 2-k}$, one has $q>X$. Then as in [9, Section 7], we have

$$
\sup _{\alpha \in \mathfrak{m}}|h(\alpha)| \ll_{\varepsilon} P^{1-\varrho(k)+\varepsilon},
$$

where

$$
\varrho(k)=\max _{s \in \mathbb{N}} \frac{1-\Delta_{s}}{4 s} .
$$

On putting $s=\left[\frac{1}{2} k(\log k+\log \log k+1)\right]+1$, from the corollary to Theorem 2.1 we obtain the bound

$$
\varrho(k) \geq(2 k(\log k+\log \log k+2+o(1)))^{-1} .
$$

Further, by the analysis of [9, Section 8], as in [14, Section 4], we have

$$
G(k) \leq \min _{v \in \mathbb{N}}\left(3+2 v+2\left[\frac{\Delta_{v}}{2 \varrho(k)}\right]\right) .
$$

But from the corollary to Theorem 2.1, we have $\Delta_{v} \leq k e^{1-2 v / k}$. On putting $v=\left[\frac{1}{2} k(\log k+\log \log k+1+\log 2)\right]+1$, we therefore obtain

$$
\Delta_{v} \leq \frac{1}{2 \log k},
$$

and hence

$$
G(k) \leq k(\log k+\log \log k+1+\log 2)+k+k \frac{\log \log k}{\log k}(1+o(1)) .
$$

This completes the proof of Theorem 1.3. 


\section{References}

[1] R. C. Baker, Diophantine Inequalities, London Math. Soc. Monographs (N.S.) 1, Clarendon Press, Oxford, 1986.

[2] R. C. Baker, J. Brüdern and G. Harman, The fractional part of $\alpha n^{k}$ for squarefree n, Quart. J. Math. Oxford (2) 42 (1991), 421-431.

[3] I. Danicic, Contributions to Number Theory, Ph.D. Thesis, London, 1957.

[4] G. Harman, Trigonometric sums over primes I, Mathematika 28 (1981), 249-254.

[5] D. R. Heath-Brown, On the fractional part of $\alpha n^{k}$, ibid. 35 (1988), 28-37.

[6] H. Heilbronn, On the distribution of the sequence $n^{2} \theta(\bmod 1)$, Quart. J. Math. Oxford 19 (1948), 249-256.

[7] K. Thanigasalam, Some new estimates for $G(k)$ in Waring's problem, Acta Arith. 42 (1982), 73-78.

[8] R. C. Vaughan, The Hardy-Littlewood Method, Cambridge Tracts Math. 80, Cambridge Univ. Press, 1981.

[9] —, A new iterative method in Waring's problem, Acta Math. 162 (1989), 1-71.

[10] - A new iterative method in Waring's problem, II, J. London Math. Soc. (2) 39 (1989), 219-230.

[11] R. C. Vaughan and T. D. Wooley, Further improvements in Waring's problem, to appear.

[12] I. M. Vinogradov, Analytischer Beweis des Satzes über die Verteilung der Bruchteile eines ganzen Polynoms, Bull. Acad. Sci. USSR (6) 21 (1927), 567-578.

[13] H. Weyl, Über die Gleichverteilung von Zahlen mod. Eins, Math. Ann. 77 (1916), 313-352.

[14] T. D. Wooley, Large improvements in Waring's problem, Ann. of Math. 135 (1992), 131-164.

DEPARTMENT OF MATHEMATICS

UNIVERSITY OF MICHIGAN

ANN ARBOR

MICHIGAN 48109-1003

U.S.A.

E-mail: TREVOR WOOLEY@MATH.LSA.UMICH.EDU 